\title{
Theory and Simulation of the Smooth Quantum Hydrodynamic Model
}

\author{
CARL L. GARDNER* \\ Department of Mathematics, Arizona State University, Tempe, AZ 85287-1804
}

(Received 13 August 1997; In final form 1 December 1998)

\begin{abstract}
The "smooth" quantum hydrodynamic (QHD) model is derived specifically to handle in a mathematically rigorous way the discontinuities in the classical potential energy which occur at heterojunction barriers in quantum semiconductor devices. Smooth QHD model simulations of the resonant tunneling diode are presented which exhibit enhanced negative differential resistance when compared with simulations using the original $O\left(\hbar^{2}\right)$ QHD model. In addition, smooth QHD simulations of a classical electron shock wave are presented which agree with classical hydrodynamic model simulations and which do not exhibit the spurious dispersive oscillations of the $O\left(\hbar^{2}\right)$ QHD model.
\end{abstract}

Keywords: Quantum hydrodynamic model, resonant tunneling diode

\section{INTRODUCTION}

An extension of the classical hydrodynamic model to include quantum transport effects was presented in Refs. [1, 2]. This "smooth" quantum hydrodynamic (QHD) model is derived specifically to handle in a mathematically rigorous way the discontinuities in the classical potential energy which occur at heterojunction barriers in quantum semiconductor devices. The model is valid to all orders of $\hbar^{2} /\left(m T_{0} l^{2}\right.$ ) (where $m$ is the electron effective mass, $T_{0}$ is the ambient temperature, and $l$ is a typical length scale for the problem) and to first order in the classical potential energy.
The smooth QHD equations have the same form as the classical hydrodynamic equations:

$$
\begin{gathered}
\frac{\partial n}{\partial t}+\frac{\partial}{\partial x_{i}}\left(n u_{i}\right)=0 \\
\frac{\partial}{\partial t}\left(m n u_{j}\right)+\frac{\partial}{\partial x_{i}}\left(m n u_{i} u_{j}-P_{i j}\right) \\
=-n \frac{\partial V}{\partial x_{j}}-\frac{m n u_{j}}{\tau_{p}} \\
\frac{\partial W}{\partial t}+\frac{\partial}{\partial x_{i}}\left(u_{i} W-u_{j} P_{i j}+q_{i}\right) \\
=-n u_{i} \frac{\partial V}{\partial x_{i}}-\frac{\left(W-(3 / 2) n T_{0}\right)}{\tau_{w}}
\end{gathered}
$$

*Tel.: 602-965-0226, Fax: 602-965-0461, e-mail: gardner@asu.edu 
where $n$ is the electron density, $u_{i}$ is the velocity, $P_{i j}$ is the stress tensor, $V$ is the potential energy, $W$ is the energy density, and $q_{i}$ is the heat flux. Indices $i, j$ equal $1,2,3$, and repeated indices are summed over. Electron scattering is modeled by the standard relaxation time approximation, with momentum and energy relaxation times $\tau_{p}$ and $\tau_{w}$.

The stress tensor and energy density are

$$
\begin{gathered}
P_{i j}=-n T \delta_{i j}-\frac{\hbar^{2} n}{4 m T} \frac{\partial^{2} \bar{V}}{\partial x_{i} \partial x_{j}} \\
W=\frac{3}{2} n T+\frac{1}{2} m n u^{2}+\frac{\hbar^{2} n}{8 m T} \nabla^{2} \bar{V}
\end{gathered}
$$

where $T$ is the temperature of the electron gas and the "quantum" potential $\bar{V}$ is given by $(\beta=1 / T)$

$$
\begin{aligned}
\bar{V}(\beta, \mathbf{x})= & \int_{0}^{\beta} \frac{d \beta^{\prime}}{\beta}\left(\frac{\beta^{\prime}}{\beta}\right)^{2} \\
& \int d^{3} x^{\prime}\left(\frac{2 m \beta}{\pi\left(\beta-\beta^{\prime}\right)\left(\beta+\beta^{\prime}\right) \hbar^{2}}\right)^{3 / 2} \\
& \times \exp \left\{-\frac{2 m \beta}{\left(\beta-\beta^{\prime}\right)\left(\beta+\beta^{\prime}\right) \hbar^{2}}\right. \\
& \left.\left(\mathbf{x}^{\prime}-\mathbf{x}\right)^{2}\right\} V\left(\mathbf{x}^{\prime}\right) .
\end{aligned}
$$

The transport equations (1)-(3) are coupled to Poisson's equation for the electrostatic potential energy

$$
\nabla \cdot\left(\varepsilon \nabla V_{P}\right)=e^{2}(N-n)
$$

where $\varepsilon$ is the dielectric constant, $e>0$ is the electronic charge, and $N$ is the density of donors. The total potential energy $V$ consists of two parts, one from Poisson's equation $V_{P}$ and the other from the potential barriers $V_{B}$ :

$$
V=V_{B}+V_{P} .
$$

$V_{B}$ has a step function discontinuity at potential barriers.

To derive the stress tensor and energy density, we constructed an effective density matrix as an $O(\beta V)$ solution to the Bloch equation. Then using the momentum-shifted effective density matrix, we took moments of the quantum Liouville equation to derive the smooth QHD equations [1].

The original $O\left(\hbar^{2}\right)$ QHD equations (see Refs. $[3,4]$ and references therein) have been remarkably successful in simulating the effects of electron tunneling through potential barriers including single and multiple regions of negative differential resistance and hysteresis in the current-voltage curves of resonant tunneling diodes $[4,5,6]$. The stress tensor and energy density for the $O\left(\hbar^{2}\right)$ model are the same as in Eqs. (4) and (5), with $\bar{V}$ replaced by its $\hbar^{2} /\left(m T l^{2}\right) \rightarrow 0$ approximation

$$
\bar{V}=\frac{V}{3}+O\left(\hbar^{2} /\left(m T l^{2}\right)\right)
$$

However in order to avoid infinite derivatives at heterojunctions, the model relies on replacing (third) derivatives of the potential with derivatives of the logarithm of the electron density using the thermal equilibrium ansatz

$$
n \sim \exp \{-\beta V\} .
$$

The thermal equilibrium ansatz breaks down for applied voltages $\Delta V$ for which $\beta \Delta V \sim 1$.

The smooth QHD equations avoid the second derivatives of a delta function that occur at heterojunction discontinuities in the $O\left(\hbar^{2}\right)$ theory (before using the ansatz (10)). In fact, the smooth QHD equations contain at worst a step function discontinuity.

To see this, we define the 1D smooth effective potential in the momentum conservation equation (2) as the most singular part of $V-P_{11}$ :

$$
U=V+\frac{\hbar^{2}}{4 m T} \frac{d^{2} \bar{V}}{d x^{2}} .
$$

As can be proved using Fourier transforms [1], the smooth effective potential is smoother by two degrees than the classical potential $V$; i.e., if $V$ has a discontinuity, then $U$ is once differentiable.

The double integration (over both space and temperature) provides sufficient smoothing so that the $P_{11}$ term in the smooth effective potential 
cancels the leading singularity in the classical potential at a barrier (see Fig. 1), leaving a residual smooth effective potential with a lower potential height in the barrier region. This cancellation and smoothing makes the barriers partially transparent to the particle flow and provides the mechanism for particle tunneling in the QHD model. Note that the effective barrier height approaches zero as $T \rightarrow 0$. This effect explains in fluid dynamical terms why particle tunneling is enhanced at low temperatures. As $T \rightarrow \infty$, the effective potential approaches the classical double barrier potential and quantum effects in the QHD model are suppressed.

Jump relations may be derived by integrating the QHD equations (1)-(3) across a wave:

$$
\begin{aligned}
& -\frac{\hbar^{2}}{4 m T}\left[\frac{d^{2} \bar{V}}{d x^{2}}\right]=[V] \\
& \kappa\left[\frac{d T}{d x}\right]=-\frac{1}{2} n u[V]
\end{aligned}
$$

where $u$ is the normal velocity and $[\chi]=\chi_{+}-\chi_{-}$is the jump in $\chi$ across the wave. Equation (12) says that the jump in the classical potential $V$ is cancelled by the jump in the quantum part of the stress tensor and Eq. (13) says that the jump in $d T / d x$ is controlled by the jump in $V$.

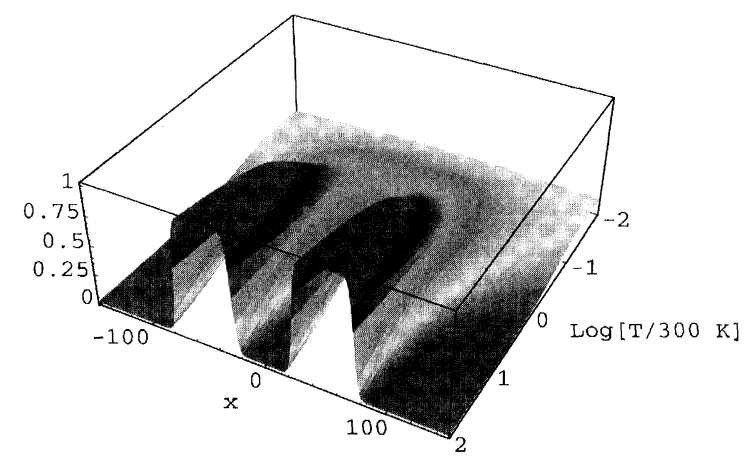

FIGURE 1 Smooth effective potential for electrons in GaAs for $50 \AA$ wide unit potential double barriers and $50 \AA$ wide well as a function of $x$ and $\log _{10}(T / 300 \mathrm{~K})$.

\section{SIMULATION OF THE RTD}

The smooth QHD transport equations are a set of hyperbolic PDEs with a parabolic heat conduction term in the energy conservation equation. Hyperbolic methods from computational gasdynamics like the ENO, piecewise parabolic, and discontinuous Galerkin methods are well suited for simulating the transient smooth QHD model. Steady-state solutions may be obtained as the asymptotic large $t$ limit.

Here we will use a simple, robust steady-state method that is an order of magnitude faster than the transient solvers in 1D. The 1D steady-state smooth QHD equations are discretized [4] using a conservative upwind method adapted from computational fluid dynamics. The discretized equations are then solved by a damped Newton method.

We present simulations of a GaAs resonant tunneling diode with $\mathrm{Al}_{x} \mathrm{Ga}_{1-x}$ As double barriers at $77 \mathrm{~K}$. The barrier height is set equal to $0.05 \mathrm{eV}$. The diode consists of $n^{+}$source (at the left) and drain (at the right) regions with the doping density $N=10^{18} \mathrm{~cm}^{-3}$, and an $n$ channel with $N=5 \times$ $10^{15} \mathrm{~cm}^{-3}$. The channel is $250 \AA$ long, the barriers are $50 \AA$ wide, and the quantum well between the barriers is $50 \AA$ wide. Note that the device has $50 \AA$ spacers between the barriers and the contacts.

Smooth QHD simulations of the resonant tunneling diode exhibit enhanced negative differential resistance when compared to simulations using the original $O\left(\hbar^{2}\right)$ QHD model. The currentvoltage curve for the resonant tunneling diode at $77 \mathrm{~K}$ is plotted in Figure 2. It is interesting that the original $O\left(\hbar^{2}\right)$ QHD model fails to produce negative differential resistance for this device. Note that the $\mathrm{I}-\mathrm{V}$ curves agree in the thermal equilibrium limit $V \rightarrow 0$. Thus we believe the original $O\left(\hbar^{2}\right)$ QHD model is only valid for applied voltages $\Delta V$ for which $\beta \Delta V \ll 1$.

\section{SIMULATION OF AN ELECTRON SHOCK WAVE}

Classical hydrodynamic model simulations of a steady-state electron shock wave [7] in a one 


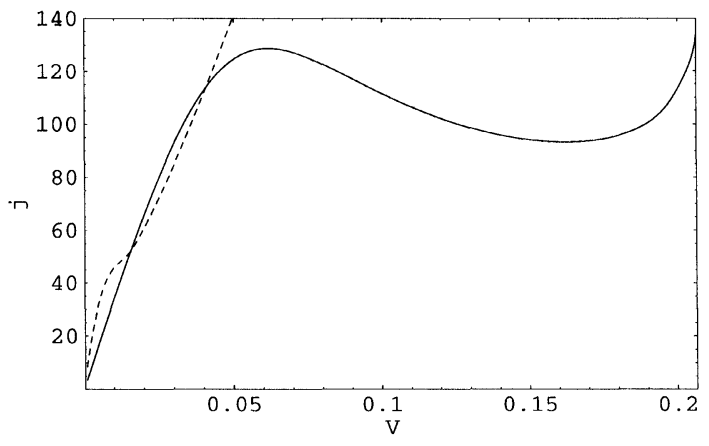

FIGURE 2 Current density in kiloamps $/ \mathrm{cm}^{2} v s$. voltage for the resonant tunneling diode at $77 \mathrm{~K}$. The solid curve is the smooth QHD computation and the dotted line is the $O\left(\hbar^{2}\right)$ computation. The barrier height is $0.05 \mathrm{eV}$.

micron Si semiconductor device at $77 \mathrm{~K}$ were validated in Ref. [8] by a Monte Carlo simulation of the Boltzmann equation using the DAMOCLES [9] program. A shock profile develops in the channel as the supersonic flow on entering the channel breaks to a subsonic flow, in analogy with gas dynamical flow in a Laval nozzle. The electron shock wave is analogous to the gas dynamical shock wave in the Laval nozzle, where the $n^{+}-n-n^{+}$doping of the diode corresponds to the converging/diverging geometry of the Laval nozzle.

The steady-state upwind shock simulations presented in Refs. [7,8] were reproduced in Ref. [10] using a time-dependent "essentially nonoscillatory" (ENO) upwind scheme, a higher-order Godunov method.

In this Section I will compare classical hydrodynamic $v s$. smooth and $O\left(\hbar^{2}\right)$ QHD simulations of an electron shock wave in a GaAs $n^{+}-n-n^{+}$ diode at $77 \mathrm{~K}$. The $n^{+}$doping density is $10^{18} \mathrm{~cm}^{-3}$ and the $n$ doping density is $10^{15} \mathrm{~cm}^{-3}$. The diode consists of a 0.1 micron source, a 0.1 micron channel, and a 0.1 micron drain.

Figure 3 presents the classical hydrodynamic model vs. smooth QHD and $O\left(\hbar^{2}\right)$ QHD model simulations with $900 \Delta x$ of an electron shock wave at a bias of $V=1$ volt. The shock profile is most clearly visible in the velocity plot Figure 3 (the

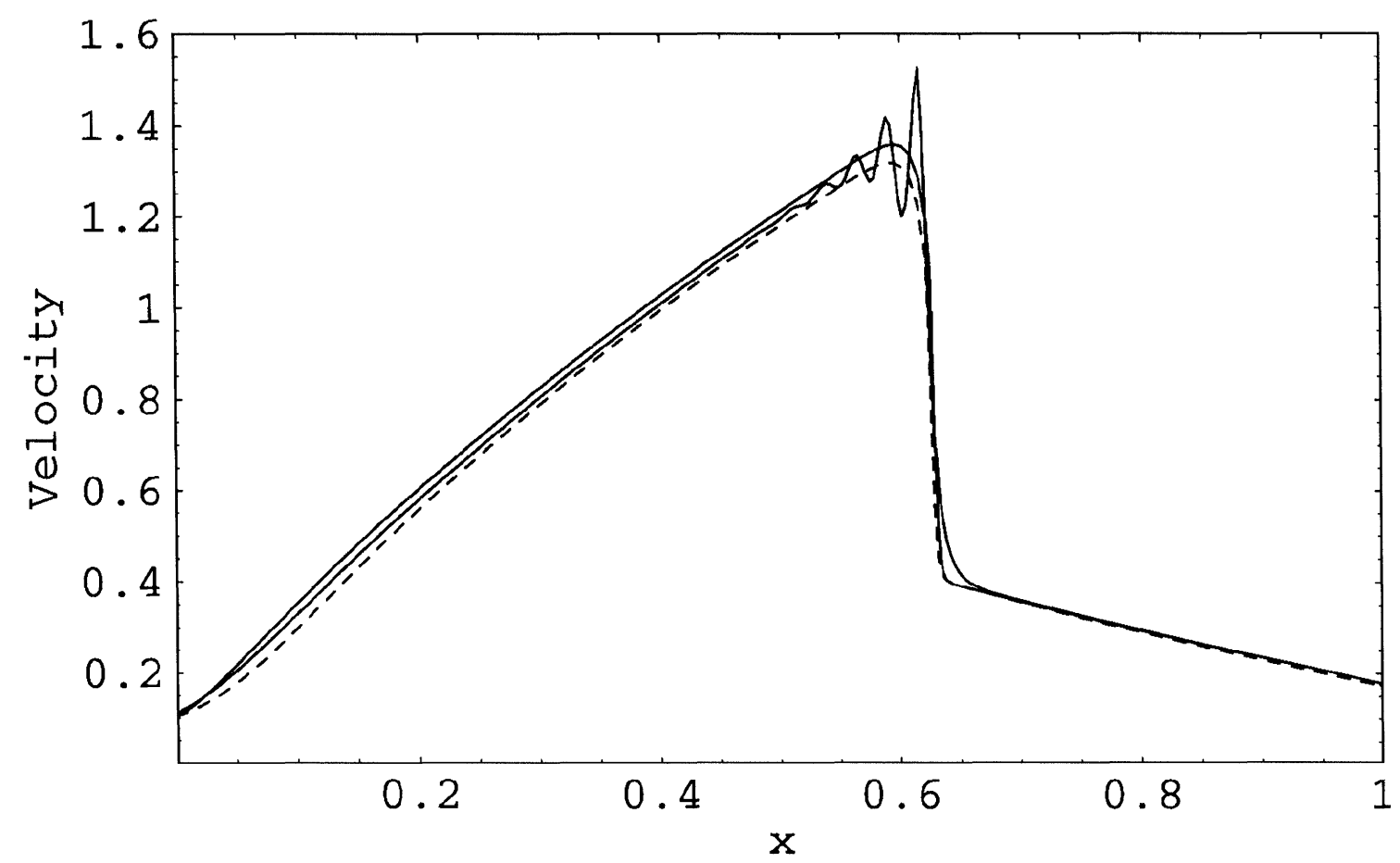

FIGURE 3 Electron velocity in $10^{8} \mathrm{~cm} / \mathrm{s}$ exhibiting an electron shock wave for the $n^{+}-n-n^{+}$diode: classical hydrodynamic (solid), smooth QHD (dotted), $O\left(\hbar^{2}\right)$ QHD (solid oscillatory). $x$ is in 0.1 microns. 
electrons flow from left to right). The flow is supersonic (Mach 12.4) at the velocity peak just inside the channel, and subsonic (Mach 0.6) at the end of the wave where the velocity makes a "bend" to the plateau in the channel. Small quantum effects primarily associated with the source/channel junction slightly lower the peak velocity of the smooth QHD simulation with respect to the classical hydrodynamic results.

The smooth QHD transport equations have four hyperbolic modes and one parabolic heat conduction mode. The original $O\left(\hbar^{2}\right)$ QHD transport equations in contrast have two hyperbolic modes, two Schrödinger modes, and one parabolic mode. The dispersive Schrödinger modes are mathematically problematic [11] for transonic flows in which the electron velocity $v$ changes from supersonic $(v>c)$ to subsonic $(v<c)$ (where $c=\sqrt{T / m}$ is the soundspeed in the electron gas), creating dispersive oscillations just before the shock wave breaks (see Pietra and Pohl's analysis). The QHD simulations presented here incorporate real heat conduction as well as numerical viscosity, so only a few oscillations are reflected in Figure 3.

\section{Acknowledgement}

Research supported in part by the National Science Foundation under grants DMS-9706792 and INT-9603253.

\section{References}

[1] Gardner, C. L. and Ringhofer, C. (1996). "Smooth quantum potential for the hydrodynamic model", Physical Review, E53, 157-167.

[2] Gardner, C. L. and Ringhofer, C. (1998). "Approximation of thermal equilibrium for quantum gases with discon- tinuous potentials and application to semiconductor devices", SIAM Journal on Applied Mathematics, 58, $780-805$.

[3] Grubin, H. L. and Kreskovsky, J. P. (1989). "Quantum moment balance equations and resonant tunnelling structures", Solid-State Electronics, 32, 1071-1075.

[4] Gardner, C. L. (1994). "The quantum hydrodynamic model for semiconductor devices", SIAM Journal on Applied Mathematics, 54, 409-427.

[5] Gardner, C. L. (1995). "Resonant tunneling in the quantum hydrodynamic model”, VLSI Design, 3, 201-210.

[6] Chen, Z., Cockburn, B., Gardner, C. L. and Jerome, J. W. (1995). "Quantum hydrodynamic simulation of hysteresis in the resonant tunneling diode", Journal of Computational Physics, 117, 274-280.

[7] Gardner, C. L. (1991). "Numerical simulation of a steadystate electron shock wave in a submicrometer semiconductor device", IEEE Transactions on Electron Devices, 38, $392-398$

[8] Gardner, C. L. (1993). "Hydrodynamic and Monte Carlo simulation of an electron shock wave in a one micrometer $n^{+}-n-n^{+}$diode", IEEE Transactions on Electron Devices, 40, 455-457.

[9] Fischetti, M. V. and Laux, S. E. (1988). "Monte Carlo analysis of electron transport in small semiconductor devices including band-structure and space-charge effects", Physical Review, B38, 9721-9745.

[10] Fatemi, E., Gardner, C. L., Jerome, J. W., Osher, S. and Rose, D. J. (1991). "Simulation of a steady-state electron shock wave in a submicron semiconductor device using high-order upwind methods", In: Computational Electronics: Semiconductor Transport and Device Simulation, pp. 27-32, Boston: Kluwer Academic Publishers.

[11] Pietra, P. and Pohl, C., "Weak limits of the quantum hydrodynamic model", VLSI Design, this volume.

\section{Author's Biography}

Carl L. Gardner is Professor of Mathematics at Arizona State University. His current research interests lie in classical and quantum semiconductor device modeling and simulation, computational fluid dynamics, and the modeling and simulation of ion transport in the channels of cellular membranes. 

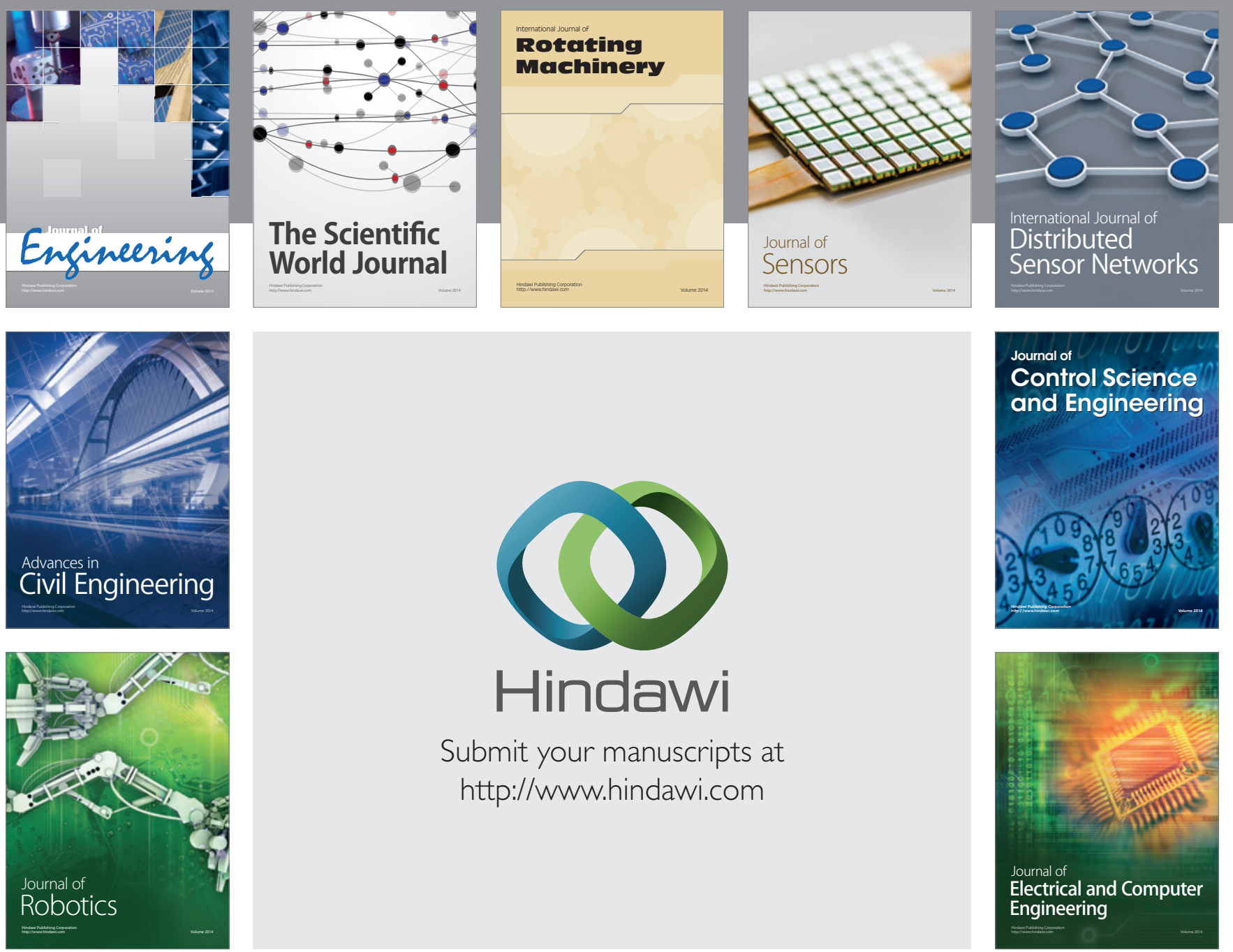

Submit your manuscripts at

http://www.hindawi.com
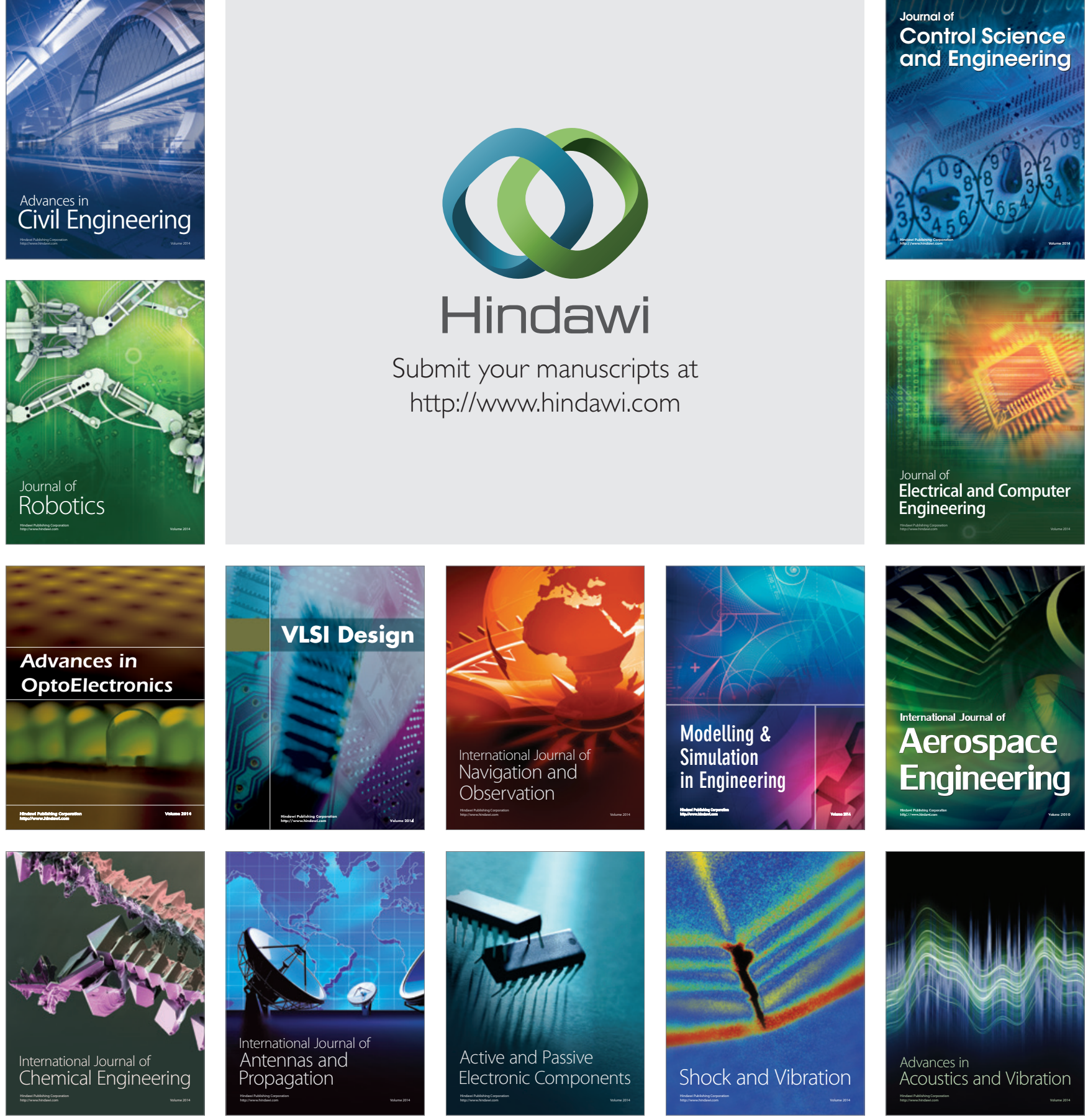